\title{
Primary cns lymphoma: current diagnosis and management
}

\begin{abstract}
Purpose: The purpose of this study was to determine our examined and evaluated the Dosimetry aspect and the early local results of cervical cancer.

Methods and Materials: Between April 2009 to January 2012, a total of 122 patients were treated with HDR Ir-192 following EBRT radiotherapy treatment, (FIGO stage IIaIIIa patients were treated with EBRT plus concomitant chemotherapy platine based, $60 \%$ of patients had radical hysterectomy after EBRT, $27 \%$ of the patients were treated with EB and were given $12 \mathrm{~Gy}$ in 2 fractions, $73 \%$ of the patients were treated with EB using 3 fractions with $7 \mathrm{~Gy}, 40 \%$ of cervical cancer patients were treated using the tandem ring applicator, while the other $60 \%$ were treated through the use of the vaginal applicator.
\end{abstract}

Results: A total of $100 \%$ of patients completed their treatment on average, 122 cases received a total of $80 \mathrm{~Gy}$ to the CTV from EBRT and Endocavitary Brachytherapy.

Conclusion: The combination of EBRT surgery and EB remains the best options of cervical cancer treatment.

Keywords: cervical cancer, brachytherapy, side effects, local relapse
Volume 4 Issue 3 - 2016

\author{
Prabhsimranjot Singh, ${ }^{\prime}$ Vivek Kumar, ${ }^{2}$ Abhinav \\ Binod Chandra ${ }^{3}$ \\ 'Fellow hematology-oncology, Maimonides Cancer Center, USA \\ ${ }^{2}$ Resident Internal medicine, Maimonides Medical Center, USA \\ ${ }^{3}$ Attending Maimonides medical center and Medical Director, \\ Yuma Regional Cancer Center, USA
}

Correspondence: Prabhsimranjot Singh, Fellow of Hematology/Oncology, Maimonides Cancer Center, 6300 Eight Avenue, 2nd Floor, Brooklyn, New York, USA, Tel 7I8-765-2600, Fax 7|8-765-2630, Email psinh2@maimonidesmed.org

Received: December 24, 2015 | Published: February II, 2016

\section{Introduction}

Primary central nervous system lymphoma (PCNSL) is a variant of non-Hodgkin's lymphoma which is confined to brain, eyes, spinal cord and/or meninges constituting 3\% of all brain tumors and $1 \%$ of all lymphomas. ${ }^{1}$ PCNSL usually affects individuals in their sixth decade of life with male preponderance. ${ }^{2}$ Immunodeficiency conditions like AIDS, iatrogenic immune-suppression for organ transplantation, congenital immune-deficiency and autoimmune conditions are predisposing risk factors. The median age of presentation in AIDS patient is 35 years and males are affected ten times more often than females. Most of the patients are affected if profoundly immunosuppressed with CD $4<100$ cells/ $\mu 1 .{ }^{3}$ PCNSL arises in fewer than $5 \%$ of organ transplant recipients with risk varying approximately $1-2 \%$ in kidney transplant patients to about $2-7 \%$ in heart, lung and liver transplant recipients. ${ }^{4}$

The incidence of PCNSL may be increasing in United States mainly in those age above 60 years old. ${ }^{1}$ Because of its rarity, there is a gap in our knowledge of its behavior, diagnosis and treatment. There has been a considerable progress in last 20 years in the understanding of tumor biology and therapeutic management options leading to dramatic improvement in outcome. ${ }^{5}$ This review highlights the key advances in our understanding of diagnosis and management of PCNSL.

\section{Clinical presentation and diagnosis}

\section{History}

Patients with PCNSL present with non-specific symptoms which frequently delays the diagnosis. When present, signs of elevated intra cranial hypertension like headache, nausea, vomiting and altered mental status (memory loss, confusion, etc.) are found in about onethird of cases. ${ }^{2} 10 \%$ cases have ocular involvement and may present with blurred vision and visual disturbances. New-onset seizures are less frequent than other forms of brain tumors because of involvement of subcortical white matter and affects fewer than $10 \%$ of cases. B-symptoms like fever, weight loss and night sweats are also rare compared to other forms of NHL. ${ }^{6}$

\section{Physical examination}

Approximately half of the patients have neurological deficits like cranial nerve palsies, hemiparesis, aphasia, and ataxia. Ocular involvement, may lead to vision loss, papilledema, optic nerve infiltration or intraocular tumor on slit lamp examination. ${ }^{2}$

\section{Blood examination}

Cytopenias or pancytopenia indicates bone marrow involvement. Preexisting renal and liver abnormalities may limit the use of methotrexate. Elevated LDH is a poor prognostic indicator in PCNSL. Other potential seromarkers for diagnosis may include Antithrombin III, Soluble CD27, interleukin-10.7 Additional laboratory tests are warranted in HIV positive patients. These include CD4 count, viral load testing (RT-PCR), and antiretroviral resistance testing.

\section{CSF examination}

CSF cytology and biochemistry is typically with in normal limits. Notably brain imaging must be done to rule out cerebellar mass lesions before lumbar puncture (LP) because LP is contraindicated in these patients due to risk of brain herniation. Promising diagnostic markers in CSF which are still in experimental stage include MicroRNAs, Antithrombin III, soluble CD 27 and Pappenheim-stained cytopsin. CSF microRNAs are a potential noninvasive biomarker for diagnosis with sensitivity and specificity of $95.7 \%$ and $96.7 \%$ respectively in preliminary studies. Antithrombin III is a promising surrogate marker of PCNSL that may be particularly helpful in lesions not amenable to biopsy. Soluble CD27 has been described as a marker of CSF involvement in PCNSL. The detection of leptomeningeal involvement in lymphoma patients is suboptimal by cytopathology alone and thus testing of immunocytochemistry with B cell markers, e.g. CD20, CD10, BCL6, and MUM1 is strongly recommended. ${ }^{7}$ 


\section{Neuroimaging}

CT imaging: CT scan of head detects up to $90 \%$ brain lesions in PCNSL patients. Lesions are usually solitary iso - to hyper dense mass lesions with ring enhancement localized to periventricular regions of brain. Edema is less prominent than with other CNS malignancies, such as glioma or metastasis. CT of chest, abdomen, and pelvis may be indicated to evaluate for metastasis in patients with B symptoms. ${ }^{8}$

MRI: MRI with or without gadolinium enhancement may detect lesions not seen on CT, as well as leptomeningeal involvement. Lesions may be hypo- to isointense with dense enhancement on gadolinium. MRI of spine is particularly indicated in work up of idiopathic uveitis because intraocular lymphoma is a consideration in these patients and up to $80 \%$ of intraocular lymphoma patients have CNS involvement. ${ }^{9}$

Brain biopsy: The definitive diagnosis of PCNSL is made on stereotactic brain biopsy. Histologically majority of tumors are diffuse large B cell lymphomas (DLBCL). Only 1 to 3\% are T-cell phenotype, and approximately $3 \%$ are low grade lymphomas of either $\mathrm{B}$ or $\mathrm{T}$ cell phenotype. ${ }^{10}$ Immunohistochemical work up requires staining for CD45 (common leucocyte antigen for both $\mathrm{B}$ and $\mathrm{T}$ cells), CD 20 (B-cells), and CD3 (T-cells). Immunostaining of GFAP identifies astrocytes and highlights the gliosis of the affected brain parenchyma. ${ }^{12}$ Proliferation markers like MIB-1 are rarely useful in guiding treatment or prognosis in PCNSL. The MIB-1 labelling index is generally greater than $50 \%$ in these tumors. ${ }^{11}$

\section{Prognosis of PCNSL}

Prognosis: Without treatment PCNSL has a dismal prognosis with median survival of 1.5 months. WBRT can prolong the survival to 10-18 months and combination chemotherapy can further increase it to beyond 40 months. ${ }^{12}$ Long term survival after methotrexate based therapy is seen in approximately $15-20 \%$ individuals. According to 'The International Extra nodal Lymphoma Study Group (IELSG)', age over 60 years, elevated LDH, Eastern Cooperative (ECOG) Group Performance Status greater than one, elevated CSF protein and tumor location in the brain (periventricular, basal ganglia, brainstem and cerebellum) are useful predictors of outcome in PCNSL patients. ${ }^{13}$ The presence of 0-1,2-3, or 4-5 adverse risk factors correlates with 2 -years survival rates of $80 \%, 48 \%$ and $15 \%$ respectively. ${ }^{14}$ Despite being the strongest risk factor there is controversy regarding the age limit above which prognosis declines. The Memorial Sloan-Kettering (MSK) prognostic index employs a limit of age 50 years instead of $60 .{ }^{15}$ In recent trials outcomes in patients age above 60 years was not worse than younger patients though it requires further validation. ${ }^{16}$

\section{Treatment}

\section{Induction chemotherapy}

High dose Methotrexate: The efficacy of Methotrexate in the treatment of Primary CNS lymphoma was first demonstrated by Ervin \& Canellos in $1980 .{ }^{17}$ The primary CNS lymphoma cells inside brain are more sensitive to MTX and thus HD-MTX forms the backbone of PCNL therapy. HD-MTX is also shown a treatment related prognostic marker for survival in these patients. ${ }^{18}$ There is no firm definition of HD-MTX and doses from $1 \mathrm{~g} / \mathrm{m}^{2}$ to $8 \mathrm{~g} / \mathrm{m}^{2}$ has been reported in clinical trials. ${ }^{19}$ Importantly Lippens and colleagues ${ }^{20}$ demonstrated that intravenous administration of Methotrexate over 3 hours (3g/ $\mathrm{m}^{2}$ ) leads to superior concentrations in cerebrospinal fluid (CSF) than infusion over 24 hours. Despite detectable meningeal disease in up to $15 \%$ patients at diagnosis, intrathecal therapy has failed to achieve significantly better outcome. ${ }^{21}$ Glantz et al demonstrated higher CSF levels after intravenous Methotrexate $\left(8 \mathrm{~g} / \mathrm{m}^{2}\right)$ than intrathecal injection with $12 \mathrm{~g} / \mathrm{m}^{2}$ Methotrexate. ${ }^{16}$ Furthermore retrospective studies failed to show any additional benefit of intrathecal therapy over HD-MTX $\left(3 \mathrm{~g} / \mathrm{m}^{2}\right) .{ }^{22}$ Recently two non-randomized studies have suggested higher rates of early relapse with omission of intrathecal therapy in comparison to otherwise identical chemotherapy regimens. ${ }^{23,24}$ The current guidelines recommends against the routine use of intrathecal therapy in patients who are candidates for HD-MTX $\left(3 \mathrm{~g} / \mathrm{m}^{2}\right)$ therapy. ${ }^{25}$ However the recommendations are controversial in the presence of established meningeal disease and clinical discretion is required.

Similar to MTX dosage, the number of chemotherapy cycles required for complete resolution are yet to be established. Abrey et al. ${ }^{26}$ described complete resolution after 4 cycles while Batchelor et al..$^{23}$ reported long term resolution at least in $20 \%$ patients after eight cycles of chemotherapy. The current consensus supports neuroimaging after 6 cycles of chemotherapy. If disease is completely resolved, treatment can be stopped after 8 cycles of chemotherapy. Further cycles can be considered in the presence of active disease. ${ }^{24}$

The acute toxicity of HD-MTX include nephrotoxicity seen in up to $5 \%$ patients due to the precipitation of Methotrexate and its 7-OH metabolite in renal tubules. ${ }^{25}$ The safe administration of HD-MTX warrants liberal hydration, urinary alkalinization, and avoidance of concomitant nephrotoxic drugs including NSAIDS and contrast agents. At least 48 hours should be spared between contrast injection and administration of Methotrexate. Carboxypeptidase -G2 (Glucapidase, FDA approved in 2012), a recombinant enzyme which hydrolyzes MTX reducing its toxic concentrations within 15 minutes of injection, can be used. ${ }^{26}$

\section{Rituximab}

Unlike in diffuse large B-cell lymphoma elsewhere Rituximab has not been useful in Primary CNS lymphoma because of poor CNS penetration[34]. It has been tested as a single agent or as part of combination therapy in PCNSL (off label use). In a study, 30 patients were treated with rituximab in combination with MTX, procarbazine, and vincristine [R-MPV]) followed by varying doses of whole brain radiation therapy (WBRT) and Cytarabine. Forty-four percent of patients achieved a CR after five or fewer cycles, and $78 \%$ after seven cycles. $^{27}$

In another retrospective, observational study on 36 patients with newly diagnosed PCNSL, six-month progression-free survival was significantly higher in the group receiving Rituximab in addition to Methotrexate (MTX) and Ifosfamide (IFO) (94.1 vs. $63.2 \%$, p=0.04) without significant difference in adverse reactions. ${ }^{28}$

\section{Combination chemotherapy}

In their pioneering study on 17 patients De Angelis and colleagues reported additional benefit of combination regimen consisting of intrathecal and high dose intravenous methotrexate (HD-MTX), thiotepa, and procarbazine, followed by whole-brain radiotherapy (WBRT) ${ }^{29}$ After chemotherapy, 7 patients $(41 \%)$ had a complete response (CR) and $7(41 \%)$ a partial response (PR). After RT, 13 (76\%) patients achieved a CR, $2(12 \%)$ a PR, and $2(12 \%)$ had disease progression. Relapse occurred in 7 (41\%) patients; median disease-free survival was 18 months. Median overall survival was 32 months. Nephrotoxicity was a minor complication, but grades 3 and 4 myelosuppression was found in $5(29 \%)$ patients. The authors concluded that procarbazine and thiotepa are potential candidates 
for incorporation into chemotherapy regimens aiming to decrease the incidence of neurotoxicity. First relapse and neurotoxicity within 2 years of diagnosis seem to be critical for predicting long-term outcomes

IELSG20, a phase II randomized control trial in primary CNS lymphoma patients, was conducted to assess the role of combined therapy, HD-MTX and cytarabine [30]. It demonstrated a superior CR rate of $46 \%$ compared to HD-MTX alone ( $18 \%$, p=0.006), with significant improvements in PFS. The 3 years OS was also prolonged but failed to reach significance. ( $46 \%$ vs. $32 \%$, p=0.07). The median disease free survival was 8 months in patients on combination chemotherapy against 4 months in patients who received HD-MTX monotherapy. Grade 3-4 hematological toxicity was more common in the methotrexate plus cytarabine group than in the methotrexate group (36 [92\%] vs $6[15 \%])$.

In their study by Thiel et al..$^{31}$ omission of whole-brain radiation therapy did not reduce survival..$^{31}$ There was modest improvement in the progression free survival with WBRT but it failed to translate into increased overall survival.

\section{Consolidation therapy}

The most promising results have been obtained with dose intensive CSF penetrant chemotherapy including Carmustine, Thiotepa, Etoposide and high dose Cytarabine in combination with autologous stem cell transplant (HDT-ASCT). Its efficacy was first demonstrated in relapsed/refractory PCNSL patients but subsequently studied as a consolidation approach for first-line therapy in selected patients. ${ }^{32,33}$

Thiotepa has been incorporated as a key agent in HDT-ASCT protocols for PCNSL because of its small size and lipophilicity facilitating efficient CNS penetration. ${ }^{34}$ Schorb et al. ${ }^{35}$ analyzed the outcomes in a cohort ( $\mathrm{n}=105$; Median age 54$)$ treated with thiotepa/ BCNU HDT-ASCT in first-line consolidation after HD-MTXbased induction therapy. ${ }^{36}$ The results were encouraging with fiveyear OS of $79 \%$ and a median PFS and OS of 85 and 121 months, respectively. HDT-ASCT-related mortality was $2.8 \%$, and $36 \%$ of patients received additional WBRT. In an intention to treat analysis, long-term follow-up data was recently reported on patients included in two prospective single-arm phase II trials involving HD-MTX and HD-Ara-C/thiotepa induction followed by carmustine/thiotepaconditioned ASCT, with $(n=30)$ or without $(n=13)$ WBRT. ${ }^{35}$ A total of 34 of 43 patients proceeded to ASCT and median OS for the whole cohort was 104 months with a five-year OS of $70 \%$. The lack of sensitivity of tumor cells to first line induction chemotherapy doesn't preclude from proceeding to HDT-ASCT. Long-term remissions have been demonstrated in patients who failed to achieve PR with HD-MTX-based induction prior to HDT-ASCT, but with inferior outcomes. ${ }^{37}$ Interestingly the results using BEAM (carmustine, etoposide, cytarabine, melphalan) regimen followed by autologous stem cell rescue have been disappointing, with a PFS of 9.3 months in a single institution study $(\mathrm{n}=14){ }^{27}$

In a multicenter prospective trial, a dose-intensive, nonmyeloablative, consolidation chemotherapy constituting 4 months of induction therapy with HD-MTX given every 2 weeks with oral temozolamide and intravenous Rituximab (MT-R) followed by high dose consolidation without WBRT was studied. The 2- year rate of PFS was. 57 and median time to progression was 4 years. Though well tolerated this regimen was associated with $>80 \%$ rate of grade 3 and 4 neutropenia. Apart from relatively high rate of early disease progression, disease control using this strategy appears at least comparable with chemoradiation protocols. However these results require further confirmation. This approach may be particularly useful in elderly or morbid patients, who are ineligible for HDT-ASCT and are at higher risk of developing neurotoxicity with WBRT. ${ }^{38}$

\section{Recurrent/ relapsing lymphoma}

There is no standard treatment approach for patients with relapsed and refractory disease. Retreatment with HD-MTX may be tried if patient responded to this treatment previously. ${ }^{39,40}$ Combination therapy is also shown to be beneficial. Mappa et al. ${ }^{41}$ reported a two-year OS of $25 \%$ in a retrospective study of salvage therapy with rituximab, ifosfamide, and etoposide for refractory or relapsed PCNSL. ${ }^{41}$ In studies where eligible patients have proceeded to HDTASCT, the use of thiotepa-based HDT-ASCT in relapsed PCNSL, resulted in 3 year OS rates of $60 \% .{ }^{42}$ WBRT has also been studied for salvage treatment and results have been encouraging in comparison to chemotherapy with equivalent PFS of 10-10.8 months, but are rarely long-lasting. Most often WBRT is employed for consolidation following salvage chemotherapy or as a palliative single-modality treatment in doses of 20-40 Gy. ${ }^{43}$

Temozolomide has been evaluated in a number of retrospective and prospective studies, with and without rituximab, demonstrating modest efficacy and some durable responses (ORR 14-53\%, oneyear OS 31-71 \%) but disappointing PFS of $<2-2.8$ months. ${ }^{44}$ Use of pemetrexed as an alternative antifolate agent has been reported in two small cohorts of heavily pre-treated patients with a median PFS of 5.7-5.8 months and one-year OS of $45 \%$ and studies addressing its efficacy are ongoing. ${ }^{45}$

\section{Radiation therapy}

Delayed neurotoxicity disfavors the use of this otherwise very effective treatment in patients with primary CNS lymphoma. Delayed neurotoxicity affects up to one-third patients and generally manifests as gait ataxia, dementia and incontinence. This is dose related and particularly affects older patients above 60 years old. ${ }^{46}$ The utility of radiotherapy is also limited by insufficient control of lymphoma and dissemination of tumor cells outside the radiation field. WBRT may be employed in patients with methotrexate resistance ${ }^{47} \mathrm{~A}$ recent systematic review, assessed outcomes of chemotherapy against combined modality treatment and suggested improvements in both survival and quality-adjusted life years after consolidation WBRT in patients $<60$ years old. ${ }^{48}$

\section{Surgery}

The diagnosis of PCNSL is established by stereotactic brain biopsy and resection of lymphoma has not been recommended in the past because of increased post-operative neurological deficits without any improvement in the outcome. However this notion was challenged currently in a recent retrospective analysis of the German PCNSL Study Group-1 (GPSG-1) which was a non-inferiority trial designed to test the impact of WBRT following upfront chemotherapy on overall survival. ${ }^{49} 13 \%$ had total and $14 \%$ had subtotal resections, and while $73 \%$ underwent stereotactic or open biopsies. Significantly more patients in surgery (gross total or subtotal) group remained in complete remission 6 months post resection compared to patients who were only biopsied ( $\mathrm{P}=003)$. Progression-free survival was significantly better following resection $(\mathrm{P}=.012)$, but without any improvement in overall survival. $(\mathrm{P}=085)$. The authors proposed consideration for surgery in patients with single lesions that might be safely resected. However current guidelines continue to recommend against surgical resection. 


\section{Conclusion}

The treatment approach in primary CNS lymphoma is changing rapidly. The combination modality promises the best option currently and cure can be anticipated in up to $50 \%$ patients. There may be a subsection of patients who may benefit from radiotherapy or surgical resection. Future research should be directed to identify such patients. There is also a significant need to identify novel bio markers which can identify primary refractive disease.

\section{Conflicts of Interest}

The authors declare no conflict of interest.

\section{Acknowledgments}

None.

\section{Funding}

None.

\section{References}

1. Villano JL, Koshy M, Shaikh H, et al. Age, gender and racial differences in incidence and survival in primary CNS lymphoma. Br J Cancer. 2011;105(9):1414-1418.

2. Josephson SA, Papanastassiou AM, Berger MS, et al. The diagnostic utility of brain biopsy procedures in patients with rapidly deteriorating neurological conditions or dementia. $J$ Neurosurg. 2007;106(1):72-75.

3. Cingolani A, Gastaldi R, Fassone L, et al. Epstein-Barr virus infection is predictive of CNS involvement in systemic AIDSrelated non-Hodgkin's lymphomas. J Clin Oncol. 2000;18(19): 3325-3330.

4. Schabet M. Epidemiology of primary CNS lymphoma. $J$ Neurooncol. 1999;43(3):199-201.

5. Fraser E, Gruenberg K, James L. Rubenstein New approaches in primary central nervous system lymphoma. Chin Clin Oncol. 2015; 4(1):11

6. Fischer L, Martus P, Weller M, et al. Meningeal dissemination in primary CNS lymphoma: prospective evaluation of 282 patients. Neurology. 2008;71(14):1102-1108

7. Roy S, Josephson SA, Fridlyand J, et al. Protein Biomarker Identification in the CSF of Patients With CNS Lymphoma. $J$ Clin Oncol. 2008;26(1):96-105.

8. Ferreri AJ, Reni M, Zoldan MC, et al. Importance of complete staging in nonHodgkin's lymphoma presenting as a cerebral mass lesion. Cancer. 1996;77(5): 827-833.

9. Chan CC, Rubenstein JL, Coupland SE, et al. Primary vitreoretinal lymphoma: a report from an International Primary Central Nervous System Lymphoma Collaborative Group symposium. Oncologist. 2011;16(11):1589-1599.

10. Commins DL. Pathology of Primary Central Nervous System Lymphoma. Neurosurg Focus. 2006;21(5): E2.

11. Aho R, Haapasalo H, Alanen K, et al. Proliferative activity and DNA index do not significantly predict survival in primary central nervous system lymphoma. J Neuropathol Exp Neurol. 1995;54: 826-832.
12. Ferreri AJ, Ciceri F, Brandes AA, et al. MATILDE chemotherapy regimen for primary CNS lymphoma: results at a median follow-up of 12 years. Neurology. 2014;82(15):1370-1373.

13. Rubenstein JL, Treseler P, O'Brien JM. Pathology and genetics of primary central nervous system and intraocular lymphoma. Hematol Oncol Clin North Am. 2005;19(4):705-717.

14. Ferreri AJ, Blay JY, Reni M, et al. Prognostic scoring system for primary CNS lymphomas: the International Extranodal Lymphoma Study Group experience. J Clin Oncol. 2003;21: 266-272.

15. Abrey LE, Ben-Porat L, Panageas KS, et al. (2006) Primary central nervous system lymphoma: the Memorial SloanKettering Cancer Center prognostic model. J Clin Oncol. 24(36):5711-5715.

16. Wieduwilt MJ, Valles F, Issa S, et al. Immunochemotherapy with intensive consolidation for primary CNS lymphoma: a pilot study and prognostic assessment by diffusion-weighted MRI. Clin Cancer Res. 2012;18(4):1146-1155.

17. Ervin T, Canellos GP. Successful treatment of recurrent primary central nervous system lymphoma with high-dose methotrexate. Cancer. 1990;45(7):1556-1557.

18. Blay JY, Conroy T, Chevreau C, et al. High-dose methotrexate for the treatment of primary cerebral lymphomas: analysis of survival and late neurologic toxicity in a retrospective series. $J$ Clin Oncol. 1998;16(3):864-871.

19. Skarin AT, Zuckerman KS, Pitman SW, et al. High-dose methotrexate with folinic acid in the treatment of advanced non-Hodgkin lymphoma including CNS involvement. Blood. 1977;50(6):1039-1047.

20. Lippens RJ, Winograd B. Methotrexate concentration levels in the cerebrospinal fluid during high-dose methotrexate infusions: an unreliable prediction. Pediatr Hematol Oncol. 1988;5(2):115-124.

21. Korfel A, Weller M, Martus P, et al. Prognostic impact of meningeal dissemination in primary CNS lymphoma (PCNSL): experience from the G-PCNSL-SG1 trial. Ann Oncol. 2012;23(9):2374-2380.

22. Glantz MJ1, Cole BF, Recht L, et al. High-dose intravenous methotrexate for patients with nonleukemic leptomeningeal cancer: is intrathecal chemotherapy necessary? J Clin Oncol. 1998;16(4):1561-1567.

23. Batchelor T, Carson K, O’Neill A, et al. Treatment of primary CNS lymphoma with methotrexate and deferred radiotherapy: A report of NABTT 96-07. J Clin Oncol. 2003;21(6):1044-1049.

24. Herrlinger U, Schabet M, Brugger W, et al. German cancer society neuro-oncology working group NOA-03 multicenter trial of single-agent high-dose methotrexate for primary central nervous system lymphoma. Ann Neurol. 2002;51(2):247-252.

25. NCCN Guideline and NCCN's. 2015.

26. Abrey LE, Moskowitz $\mathrm{CH}$, Mason WP, et al. Intensive methotrexate and cytarabine followed by high-dose chemotherapy with autologous stem-cell rescue in patients with newly diagnosed primary CNS lymphoma: an intent-totreat analysis. J Clin Oncol. 2003;21(22):4151-4156. 
27. Shah GD, Yahalom J, Correa DD, et al. Combined immunochemotherapy with reduced whole-brain radiotherapy for newly diagnosed primary CNS lymphoma. $J$ Clin Oncol. 2007;25(30):4730-4735.

28. Birnbaum T, Stadler EA, von Baumgarten L, et al. Rituximab significantly improves complete response rate in patients with primary CNS lymphoma. J Neurooncol. 2012;109(2):285-291.

29. DeAngelis LM, Seiferheld W, Schold SC, et al. Combination chemotherapy and radiotherapy for primary central nervous system lymphoma: Radiation Therapy Oncology Group Study. $J$ Clin Oncol. 2002;20(24):4643-464.

30. Ferreri AJ, Reni M, Foppoli M, et al. International Extranodal Lymphoma Study Group (IELSG). High-dose cytarabine plus high-dose methotrexate versus high-dose methotrexate alone in patients with primary CNS lymphoma: a randomised phase 2 trial. Lancet 2009;374(9700):1512-1520.

31. Thiel E, Korfel A, Martus P, et al. High-dose methotrexate with or without whole brain radiotherapy for primary CNS lymphoma (G-PCNSL-SG-1): a phase 3, randomised, non-inferiority trial. Lancet Oncol. 2010;11(11):1036-1047.

32. Soussain C, Hoang-Xuan K, Taillandier L, et al. Intensive chemotherapy followed by hematopoietic stem-cell rescue for refractory and recurrent primary CNS and intraocular lymphoma: Société Française de Greffe de Moëlle OsseuseThérapie Cellulaire. J Clin Oncol. 2008;26(15):2512-2518.

33. Illerhaus G, Marks R, Ihorst G, et al. High-dose chemotherapy with autologous stem-cell transplantation and hyperfractionated radiotherapy as first-line treatment of primary CNS lymphoma. J Clin Oncol. 2006;24(24):3865-3870.

34. Phillips EH, Fox CP, Cwynarski K. Primary CNS Lymphoma Curr Hematol Malig Rep. 2014;9(3):243-253.

35. Kasenda B, Schorb E, Fritsch K, et al. Prognosis after high-dose chemotherapy followed by autologous stem-cell transplantation as first-line treatment in primary CNS lymphoma-a long-term follow-up study. Ann Oncol. 2012;23(10):2670-2675.

36. Korfel A, Elter T, Thiel E, et al. Phase II study of central nervous system (CNS)-directed chemotherapy including highdose chemotherapy with autologous stem cell transplantation for CNS relapse of aggressive lymphomas. Haematologica. 2013;98(3):364-370

37. Soussain C, Choquet S, Fourme E, et al. Intensive chemotherapy with thiotepa, busulfan and cyclophosphamide and hematopoietic stem cell rescue in relapsed or refractory primary central nervous system lymphoma and intraocular lymphoma: a retrospective study of 79 cases. Haematologica. 2012;97(11):1751-1756.
38. Schorb E, Kasenda B, Atta J, et al. Prognosis of patients with primary central nervous system lymphoma after high-dose chemotherapy followed by autologous stem cell transplantation. Haematologica. 2013;98:765-770.

39. Rubenstein JL, Hsi ED, Johnson JL, et al. Intensive Chemotherapy and Immunotherapy in Patients With Newly Diagnosed Primary CNS Lymphoma: CALGB 50202 (Alliance 50202). J Clin Oncol. 31(25):3061-3068.

40. Plotkin SR, Betensky RA, Hochberg FH, et al. Treatment of relapsed central nervous system lymphoma with high-dose methotrexate. Clin Cancer Re. 2004;10(17):5643.

41. Mappa S, Marturano E, Licata G, et al. Salvage chemoimmunotherapy with rituximab, ifosfamide and etoposide (R-IE regimen) in patients with primary CNS lymphoma relapsed or refractory to high-dose methotrexate-based chemotherapy. Hematol Oncol. 2013;31(3):143-150.

42. Soussain C, Suzan F, Hoang-Xuan K, et al. Results of intensive chemotherapy followed by hematopoietic stem-cell rescue in 22 patients with refractory or recurrent primary CNS lymphoma or intraocular lymphoma. J Clin Oncol. 2001;19(3):742-749.

43. Nguyen PL, Chakravarti A, Finkelstein DM, et al. Results of whole-brain radiation as salvage of methotrexate failure for immunocompetent patients with primary CNS lymphoma. $J$ Clin Oncol. 2005;23(7):1507.

44. Reni M, Zaja F, Mason W, et al. Temozolomide as salvage treatment in primary brain lymphomas. Br J Cancer. 2007;96(6): 864-867.

45. Raizer JJ, Rademaker A, Evens AM, et al. Pemetrexed in the treatment of relapsed/refractory primary central nervous system lymphoma. Cancer. 2012;118(15):3743-3748.

46. Abrey LE, DeAngelis LM, Yahalom J. Long-term survival in primary CNS lymphoma. J Clin Oncol. 1998;16(3): 859-863.

47. Nelson DF, Martz KL, Bonner H, et al. NonHodgkin's lymphoma of the brain: can high dose, large volume radiation therapy improve survival? Report on a prospective trial by the Radiation Therapy Oncology Group (RTOG): RTOG 8315. Int $J$ Radiat Oncol Biol Phys. 1992;23(1):9-17.

48. Rica A, Chan K, Cheung MC. Combined modality therapy versus chemotherapy alone as an induction regimen for primary central nervous system lymphoma: a decision analysis. $\mathrm{Br} J$ Haematol. 2012;158(5):600-607.

49. Weller M, Martus P, Roth P, et al. German PCNSL Study Group 19.Surgery for primary CNS lymphoma? Challenging a paradigm. Neuro Oncol. 2012;14(12):1481-1484. 